\title{
Phenotypic and Molecular Investigations on Hypovirulent Cryphonectria parasitica in Italy
}

Sergio Murolo, ${ }^{\dagger}$ Department of Agricultural, Food and Environmental Sciences, Marche Polytechnic University, Ancona, Italy; Rita Milvia De Miccolis Angelini and Francesco Faretra, Department of Soil, Plant and Food Sciences, University of Bari, Italy; and Gianfranco Romanazzi, Department of Agricultural, Food and Environmental Sciences, Marche Polytechnic University, Ancona, Italy

\begin{abstract}
Chestnut blight is caused by the fungus Cryphonectria parasitica. As one of the most ecologically important diseases of Castanea spp., C. parasitica can rapidly kill trees. In Europe, mitigation of disease severity took place spontaneously through colonization of $C$. parasitica by mycoviruses, which reduced the virulence of the fungus. In the framework of a survey, 138 C. parasitica isolates were identified, and virulent/ hypovirulent phenotypes were determined through morphological properties and pathogenicity tests. For a pool of four hypovirulent isolates, dsRNA was extracted, cDNA synthesized, and a library subjected to next-generation sequencing. The bioinformatics analysis allowed

detecting and reconstructing the complete genome of Cryphonectria hypovirus 1 (CHV-1), denoted as CHV-1 Marche. When compared with the available genomes of other hypoviruses that affected the virulence of $C$. parasitica, available in databases, CHV-1 Marche showed some nucleotide diversity. The approach used in this study was effective to explore the virome inside a pool of hypovirulent $C$. parasitica isolates. Next-generation sequencing allowed us to exclude the presence of any other ssRNA and dsRNA viruses infecting the fungus and determine CHV-1 as the only responsible of hypovirulence of C. parasitica in the analyzed samples.
\end{abstract}

One of the most important and historical diseases of Castanea spp. is chestnut blight, which is caused by the Ascomycota fungus Cryphonectria parasitica (Murr) Barr (Rigling and Prospero 2017). This disease was first described in New York at the beginning of the last century. Then in Europe, C. parasitica was initially reported in 1938 in the Liguria region of Italy (Biraghi 1946), yet different introduction events were hypothesized to contribute to the current population structure as described by Dutech et al. (2010). Both in Italy and other areas in Europe, chestnut blight disease is less severe than for North America (Robin and Heiniger 2001), in part because the European chestnut (C. sativa Mill.) is less susceptible than $C$. dentata, and in part due to a reduction in the virulence of $C$. parasitica, a phenomenon deeply studied by several researchers (Grente 1965; Heiniger and Rigling 1994; MacDonald and Fullbright 1991; Milgroom and Cortesi 2004; Nuss 1992). Recovery from chestnut blight has been observed in many European countries and in some areas of the United States, such as in Michigan (Milgroom and Cortesi 2004). Particularly in Europe, this recovery was ascribed to the presence of hypovirulent strains of $C$. parasitica, which was infected by Cryphonectria hypovirus 1 (CHV-1) (Robin and Heiniger 2001). This prevalence of $\mathrm{CHV}-1$ and the consequent induced hypovirulence of $C$. parasitica have most likely prevented the occurrence of devastating chestnut blight epidemics and the destruction of the European chestnut forests to date.

$\mathrm{CHV}-1$ is a non-encapsidated cytoplasmic virus of the Hypoviridae family (Ghabrial et al. 2015). The phylogeny and time estimates suggest that CHV-1 was introduced into Italy together with $C$. parasitica, and spread across southern-central Europe and then eastern Europe (Bryner et al. 2012), due to the low genetic diversity of the C. parasitica populations. Indeed, $\mathrm{CHV}-1$ can be horizontally transmitted through hyphal anastomosis, which can occur efficiently between individual fungi that belong to the same vegetative compatibility group, but transmission can sometimes occur between genetically

${ }^{\dagger}$ Corresponding author: Sergio Murolo; E-mail: s.murolo@univpm.it

*The $\boldsymbol{e}$-Xtra logo stands for "electronic extra" and indicates that one supplementary table is published online.

Accepted for publication 2 October 2017.

() 2018 The American Phytopathological Society close vegetative compatibility types (Liu and Milgroom 1996). In the European regions where this natural hypovirulence did not appear, rapid dissemination of $\mathrm{CHV}-1$ was further aided by active biocontrol efforts (Milgroom and Cortesi 2004). To date, chestnut blight incidence in Europe is high, but due to this hypovirulence, the disease is maintained at low severity in most regions (Bryner et al. 2012, 2014; Milgroom and Cortesi 2004).

CHV-1 infection leads to superficial swollen cankers (passive cankers), with reduced fungal growth and less sporulation compared with active cankers (Milgroom and Cortesi 2004). However, a lack of any clear relationship between canker morphology and virus infection for $C$. parasitica was recently reported (Bryner et al. 2014). Hypovirus-infected $C$. parasitica isolates are often white in color, and show reduced sporulation on potato dextrose agar (PDA) growth medium compared with noninfected isolates (Milgroom and Cortesi 2004). Recently, however, blight damage recurrence has been reported for different European chestnut areas that are highly infested by the chestnut gall wasp (Dryocosmus kuriphilus), even in the presence of hypovirulent cankers (Meyer et al. 2015).

Starting from the evaluation of hypovirulent isolates of $C$. parasitica recovered from chestnut areas in the Marche and Abruzzi regions of Italy, where the damage of chestnut blight has been particularly mitigated, we applied next generation sequencing to investigate ssRNA and dsRNA mycoviruses and in particular to reconstruct the CHV-1 full-length genome, mainly involved in the hypovirulence.

\section{Materials and Methods}

Isolation and morphological characterization. Within the framework of surveys carried out from 2013 to 2016 to determine the spread and severity of chestnut blight in the Marche and Abruzzi regions of Italy, cortical tissue from plants showing "active" (eight samples) and "passive" cankers (seven samples) were collected for each site, from plants approximately $10 \mathrm{~m}$ apart from each other.

Five bark samples per canker (i.e., one in the center, four along the margins) were removed with a cork borer ( $5 \mathrm{~mm}$ diameter) that was sterilized with $95 \%$ ethanol solution during canker sampling. The bark disks were divided into two parts, which were placed into Petri dishes $(50 \mathrm{~mm}$ diameter; two pieces/plate) containing water agar (Duchefa Biochimie, Haarlem, The Netherlands), and incubated for 3 days at $25 \pm 1{ }^{\circ} \mathrm{C}$ in the dark. From the margin of each of the 
resulting fungal colonies, mycelial tips were collected and placed in Petri dishes containing PDA (Duchefa Biochimie), with further subculturing in the same medium to obtain pure colonies. Each fungal isolate was stored in a glass tube containing PDA at $4^{\circ} \mathrm{C}$.

To characterize the morphological properties of each colony of C. parasitica, they were transferred to PDA Petri dishes ( $90 \mathrm{~mm}$ diameter) and incubated for 7 days at $25 \pm 1{ }^{\circ} \mathrm{C}$ in the dark. Fungal cultures were subsequently exposed to a photoperiod of $12 \mathrm{~h}$ for an additional 7 days, again at $25 \pm 1{ }^{\circ} \mathrm{C}$. These conditions are suitable for morphological characterization of the C. parasitica isolates, and for distinguishing virulent colonies (orange mycelia, abundant pycnidia) from hypovirulent colonies (white mycelia, few or no pycnidia production) (Bissegger et al. 1997; Milgroom and Cortesi 2004).

Pathogenicity tests on chestnut cuttings. Based on the morphological characterization, $26 \mathrm{C}$. parasitica isolates (seven with orange and 19 with white mycelium) representative of the surveyed areas were selected and tested for pathogenicity on excised dormant chestnut stems. The virulence of each isolate was determined on stems (40 to $60 \mathrm{~mm}$ diameter) from healthy $C$. sativa plants. The stems were cut into 50-cm-long segments and surface disinfected with an aqueous solution of $70 \%$ ethanol. The bark of the chestnut cuttings was removed every $15 \mathrm{~cm}$ using a cork borer (5 $\mathrm{mm}$ diameter), and each wound was inoculated with a $C$. parasitica mycelial disc (5 mm diameter), then sealed with Parafilm. As a negative control, we used noninoculated PDA disks ( $5 \mathrm{~mm}$ diameter). Three isolates were assayed per cutting, with three replicates for each set of isolates tested. Both ends of each cutting were covered with cottonwool soaked with water, and incubated in a plastic box at $25^{\circ} \mathrm{C}$ under a 16 -h photoperiod for 10 days. The length and width of the necrotic areas of the cortical tissue and the wood were then measured with digital calipers (model 500-196-30; Mitutoyo America Corporation, Kanagawa, Japan) and the elliptical necrotic areas were calculated.

The means and standard deviations (sd) were calculated. These data were analyzed statistically using one-way ANOVA, and the means were compared using Duncan's multiple range tests, at $P \leq$ 0.05, using the Statistica package (Statsoft Inc., Tulsa, OK).

Reisolations of $C$. parasitica were carried out directly from necrotic lesions of cortical and wood tissue, according to the procedure already mentioned in the previous paragraph.

DNA extraction and molecular characterization of representative hypovirulent isolates. Based on morphological properties, pathogenicity, origin, and year of collection, four hypovirulent isolates (i.e., P16_2, P29B, L25A, L2A) were selected for total DNA extraction, according to Varanda et al. (2016). The DNA was used in PCR reactions with the ITS1/ITS4 primers (White et al. 1990), and sequenced at the Beckman Coulter Facility (Essex, U.K.). The raw sequences were analyzed by BLAST searches to look for sequence homology with other nucleotide sequences of $C$. parasitica available in GenBank (National Center for Biotechnology Information; NCBI). The vegetative compatibility of the selected isolates was also molecularly assessed according to Short et al. (2015).

dsRNA extraction. The same isolates of $C$. parasitica that were selected for total DNA extraction (i.e., P16_2, P29B, L25A, L2A) were tested for dsRNA. The isolates were inoculated in $40 \mathrm{ml}$ potato dextrose broth (Duchefa Biochimie) at $25^{\circ} \mathrm{C}$ for 10 days under agitation $(150 \mathrm{rpm})$. The mycelia were collected by centrifugation at $3,840 \times g$ for $15 \mathrm{~min}$, pressed between sterile sheets of paper for $24 \mathrm{~h}$, and $10 \mathrm{~g}$ for each isolate were pulverized in liquid nitrogen. The dsRNA extraction was carried out according to Al Rwahnih et al. (2009). The dsRNA extracted was treated with $25 \mathrm{ng} / \mathrm{ml}$ RNase (Sigma Aldrich, Saint Louis, MO), $1 \mathrm{mg} / \mathrm{ml}$ DNase (Promega, Madison, WI), and $5 \mathrm{mg} / \mathrm{ml}$ proteinase $\mathrm{K}$ (Sigma Aldrich), to remove ssRNA, DNA, and protein, respectively. Aliquots of the purified dsRNA were run on $1 \%$ agarose gels, and images were acquired (Gel Doc XR; Bio-Rad, Hercules, CA).

Next-generation sequencing. Deep-sequencing of the extracted dsRNA samples was performed using Illumina technology. For library preparation, $800 \mathrm{ng}$ dsRNA was subjected to heat denaturation $\left(94^{\circ} \mathrm{C}\right.$ for $\left.12 \mathrm{~min}\right)$, and flash cooled in iced water. The fragmentation, conversion to cDNA, and preparation for sequencing was performed using TruSeq RNA Sample Prep kit v2 (Illumina, San Diego, CA).

After PCR enrichment (15 cycles), the adapter-ligated cDNA fragments were quantified, diluted to $12 \mathrm{pM}$, and hybridized to a flow cell for Illumina sequencing. Clusters on the flow cell were then generated using TruSeq SR Cluster Kit v3-cBot-HS (Illumina). The flow cells with the DNA clusters were subjected to sequencing using the Illumina platform (HiScanSQ System, SELGE Network Sequencing Service; http://www.selge.uniba.it/), using SBS Sequencing v3 kit to generate 50-bp single-end reads.

Bioinformatics analysis and reconstruction of the CHV genome. High-quality (quality score $\geq 30$ ) sequenced reads were filtered using Illumina Real-Time Analysis (RTA 1.13.48) and de novo assembled using CLC Genomics Workbench 7.0.3 (CLC Bio, Qiagen, Hilden, Germany) with standard parameters, and Velvet 1.1.06, followed by Oases 0.2 .8 with a minimum contig length of $100 \mathrm{nt}$, and a multiple K-mer approach (Zhao et al. 2011). SeqMan Pro (Lasergene 10.1; DNASTAR, Inc., Madison, WI) was used for merging the resulting contigs. To search for homology to viral sequences, consensus sequences were subjected to BLASTN and BLASTX analysis against the NCBI database (www.ncbi.nlm.nih.gov), with the default parameters and the E-value cut-off of $10^{-3}$. The contigs were then classified according to the sequences with the highest bit score. Reference sequences were identified based on the BLAST results, and used in the subsequent analyses.

Transdecoder (https://transdecoder.github.io/) was used to identify open reading frames (ORFs) and to select the best assembly for the putative mycoviral sequences. Subsequently, sequencing reads were mapped back to the reference sequences of $\mathrm{CHV}-1$ and the de novo assembled viral contigs, using SeqMan NGen and SeqMan Pro (Lasergene v.10.1; DNASTAR), to determine and correct missassembly errors, and to detect single nucleotide polymorphisms (SNPs).

Phylogenetic analysis. The consensus sequence identified as CHV-1 Marche was set up in FASTA format and aligned with the Clustal X software (Thompson et al. 1997), together with the nucleotide sequences of isolates CHV-1/EP-721 (GenBank accession no. DQ861913), CHV-1/EP713 (NC_001492), CHV-1/Euro7 (AF082191), and CHV-1/CN280 (KT726153). The phylogenetic tree was built using neighbor-joining in Molecular Evolution Genetic Analysis version 5 (MEGA5) (Tamura et al. 2011). The robustness of the tree topology was verified considering the bootstrap analysis (1,000 replicates). In the phylogenetic analysis, other hypoviruses that can infect $C$. parasitica were considered as outgroups, in particular CHV-2 (NC_003534), CHV-3 (NC_000960), and CHV-4 (NC_006431), the complete genomes of which are available in the NCBI database.

\section{Results}

Morphological characterization. From 150 samples of bark showing symptoms and signs of chestnut blight and collected in the framework of the surveys, 138 colonies of $C$. parasitica were recovered (Table 1). Using PDA medium with a 12-h photoperiod at $25^{\circ} \mathrm{C}$, the majority of these isolates ( 85 out of 138 ) had orange mycelia and abundant pycnidia, and were thus recognized as virulent colonies (Fig. 1A). Fifty-three colonies grew with white mycelia and only sporadic pycnidia in the central part of the colonies, and were thus identified as hypovirulent (Fig. 1B).

Pathogenicity tests on chestnut cuttings. Significant differences were recorded between the $C$. parasitica virulent (orange) and hypovirulent isolates (white). The virulent isolates P4C4, P21A, P35D, and PD1 produced the widest elliptical necrotic areas on both the bark and the wood (Table 2). Conversely, the hypovirulent isolates showed generally slow growth and smaller necrotic areas (Table 2). After 7 days, from the reisolations of necrotic areas we obtained the corresponding virulent or hypovirulent isolates previously inoculated on the cuttings.

Molecular characterization of representative hypovirulent isolates. The DNA extraction of the four selected hypovirulent C. parasitica isolates, P16_2, P29B, L25A, and L2A, and the following 
amplification with the ITS1/ITS4 primer pair yielded a specific band of $\sim 600 \mathrm{bp}$, which showed high nucleotide homology with $C$. parasitica (GenBank accession no. KP824756). These four $C$. parasitica isolates belong to four different vegetative compatibility groups. Using multilocus PCR assays to assess vic genotype, P16_2 was confirmed molecularly as the vegetative compatibility genotype 111211 (corresponding to EU12), P29B as 2112-11 (EU17), L25A as 2211-22 (EU5), and L2A as 2112-22 (EU2).

dsRNA extraction. The dsRNA was extracted from a pool of the same four C. parasitica isolates, P16_2, P29B, L25A, and L2A, which showed similar morphologies and virulence but that were isolated from different locations. Only one band of 12,000 bp in size corresponding to L-dsRNA was obtained. No bands were visualized on the gels for P4C4, P21A, P35D, and PD1, representative virulent isolates of $C$. parasitica that were analyzed.

Sequencing and bioinformatics analysis of mycoviruses. The dsRNA was prepared for cDNA synthesis and library construction, followed by sequencing. A total of 19,068,188 short reads were initially generated in this study, which resulted in $\sim 1 \mathrm{~Gb}$ of raw sequencing data (submitted in the NCBI Sequence Read Archive [SRA] database with submission number SUB2316937, under the accession number SRR5179114). There were 626,078 nonredundant reads. After filtering, only the high-quality reads (quality score $\geq 30$ ) were considered for further analysis. To detect and identify putative mycoviral sequences in the selected isolates of $C$. parasitica, the reads were submitted to de novo assembly and analysis of the assembled contigs. CLC Genomics Workbench assembler produced 239 contigs, which included many small sequences of $<1 \mathrm{~kb}$ in length. Among the largest contigs, four that were $619,2,015,3,251$, and 6,233 nt in length together contained $98.6 \%$ of the assembled reads and were identified as partial nonoverlapping sequences of the Cryphonectria hypovirus 1 (CHV-1) genome (12,724 nts; GenBank accession no. DQ861913). Velvet, followed by Oases for the de novo RNA-Seq assembly were used to assemble the complete viral genome from the reads into a single contig. Most of the contigs produced at different $\mathrm{k}$-mer values (i.e., 37-21), which ranged between 451 and 591, were clearly overlapping and showing only small differences in their sequences.

The largest assembled sequence $(13,013 \mathrm{bp})$ that shared the highest sequence identity with the $\mathrm{CHV}-1$ genome and the best candidate coding regions within its sequence was selected as the template for realignment of the reads and for manual curation. The complete viral genome of the $\mathrm{CHV}-1$ that infects $C$. parasitica isolates from the Marche region in Italy (CHV-1_Marche) was conclusively obtained and has been submitted to GenBank (accession no. KY471627). This is $12,735 \mathrm{nt}$ in length, has a sequence identity of $98 \%$ with CHV-1 (i.e., DQ861913, AF082191) (E-value =0.0), and contains two putative ORFs of 622 and 3,164 amino acids, both with $99 \%$ amino acid identity to the two polyproteins identified as ORFA (ABI64295.1) and ORFB (ABI64296.1), respectively, in CHV-1. A total of $18,664,706$ viral reads ( $97.9 \%$ of the total reads) was mapped to the genome consensus sequence, with an average coverage of $82,223 \times$. The read depth was enough to obtain some preliminary data on the viral diversity in the analyzed $\mathrm{CHV}-1$ population from this pool of four C. parasitica isolates. Single nucleotide variants were

Table 1. Morphological features associated with virulent (V) and hypovirulent (H) Cryphonectria parasitica isolates collected during the surveys from 2013 to 2016

\begin{tabular}{|c|c|c|c|c|c|}
\hline Region & Location & Isolates & $\begin{array}{c}\text { No. of } \\
\text { isolates }\end{array}$ & $\begin{array}{c}\text { Morphological features associated } \\
\text { with } \mathrm{V} \text { and } \mathrm{H} \text { C. parasitica }\end{array}$ & $\begin{array}{c}\text { Year of } \\
\text { collection }\end{array}$ \\
\hline \multirow[t]{26}{*}{ Marche } & \multirow[t]{3}{*}{ Montemonaco (AP) } & $\begin{array}{l}\text { P1, P2, P3, P4, P4C4, P7, P8, P9, P11, } \\
\text { P13b }\end{array}$ & 12 & $\mathrm{~V}$ & 2013 \\
\hline & & PA2, PD1 & & & \\
\hline & & $\begin{array}{l}\text { P3B, P4C2, P5, P6D, P6C, P10C, } \\
\text { P11C, P12, P13A, PA1, PC }\end{array}$ & 11 & $\mathrm{H}$ & \\
\hline & \multirow[t]{2}{*}{ Rotella (AP) } & $\mathrm{P} 14, \mathrm{P} 15, \mathrm{P} 16, \mathrm{P} 17, \mathrm{P} 18, \mathrm{P} 19 \mathrm{C}$ & 6 & $\mathrm{~V}$ & 2013 \\
\hline & & P14-1, P16_2, P19A, P20 & 4 & $\mathrm{H}$ & \\
\hline & \multirow{2}{*}{ Colle San Marco (AP) } & $\mathrm{P} 40, \mathrm{P} 41, \mathrm{P} 42, \mathrm{P} 44$ & 4 & $\mathrm{~V}$ & 2013 \\
\hline & & $\mathrm{P} 40 \mathrm{~B}, \mathrm{P} 43$ & 2 & $\mathrm{H}$ & \\
\hline & \multirow[t]{2}{*}{ Colle, Ascoli Piceno } & L24b, L24a, L26A & 3 & V & 2015 \\
\hline & & L25A, L26B & 2 & $\mathrm{H}$ & \\
\hline & \multirow{2}{*}{ San Gregorio, Ascoli Piceno } & D3A, D4A & 2 & $\mathrm{~V}$ & 2016 \\
\hline & & D1A, D2B, D6B, D9B, D4B, L2A & 6 & $\mathrm{H}$ & \\
\hline & \multirow[t]{2}{*}{ Montefortino (AP) } & $\mathrm{P} 29 \mathrm{c}, \mathrm{P} 30, \mathrm{P} 31 \mathrm{~A}$ & 3 & $\mathrm{~V}$ & 2013 \\
\hline & & $\mathrm{P} 28 \mathrm{~b}, \mathrm{P} 29 \mathrm{~B}, \mathrm{P} 30 \mathrm{D}$ & 3 & $\mathrm{H}$ & \\
\hline & \multirow{2}{*}{$\begin{array}{l}\text { Collefalciano, Acquasanta Terme } \\
\text { (AP) }\end{array}$} & $\mathrm{P} 21 \mathrm{~A}, \mathrm{P} 22 \mathrm{~B}, \mathrm{P} 23, \mathrm{P} 25 \mathrm{~B}, \mathrm{P} 26, \mathrm{P} 27$ & 6 & $\mathrm{~V}$ & 2013 \\
\hline & & $\mathrm{P} 21 \mathrm{C}, \mathrm{P} 22 \mathrm{~A}, \mathrm{P} 24, \mathrm{P} 25$ & 4 & $\mathrm{H}$ & \\
\hline & \multirow[t]{3}{*}{ Pozza, Acquasanta Terme (AP) } & L16A, L16b, L17A, L17b, L18b, L18 & 9 & $\mathrm{~V}$ & 2015 \\
\hline & & E4A, E6A, E8 B & & & 2016 \\
\hline & & $\mathrm{E} 2 \mathrm{~B}, \mathrm{E} 3 \mathrm{~B}, \mathrm{E} 7 \mathrm{~B}$ bis & 3 & $\mathrm{H}$ & 2016 \\
\hline & \multirow[t]{4}{*}{ Umito, Acquasanta Terme (AP) } & L19a, L19B, L20, L21a & 9 & $\mathrm{~V}$ & 2015 \\
\hline & & $\mathrm{A} 3 \mathrm{~A}, \mathrm{~A} 4 \mathrm{~B}, \mathrm{~A} 8 \mathrm{~A}, \mathrm{~A} 9 \mathrm{~A}, \mathrm{~A} 15 \mathrm{~B}$ & & & 2016 \\
\hline & & L21b, L22A, L22b & 8 & $\mathrm{H}$ & 2015 \\
\hline & & $\mathrm{A} 2 \mathrm{~A}, \mathrm{~A} 5 \mathrm{C}, \mathrm{A} 6 \mathrm{~A}, \mathrm{~A} 7 \mathrm{~A}, \mathrm{~A} 10 \mathrm{~B}$ & & & 2016 \\
\hline & Colle Frattale, & L27a, L28A, L29a & 3 & $\mathrm{~V}$ & 2015 \\
\hline & Acquasanta Terme (AP) & $\mathrm{L} 28 \mathrm{c}$ & 1 & $\mathrm{H}$ & \\
\hline & Rocca di Montecalvo, Acquasanta & $\mathrm{C} 5 \mathrm{~B}, \mathrm{C} 6 \mathrm{~A}$ & 2 & $\mathrm{~V}$ & 2016 \\
\hline & Terme (AP) & $\mathrm{C} 3 \mathrm{~B}, \mathrm{C} 4 \mathrm{~A}$ & 2 & $\mathrm{H}$ & \\
\hline \multirow[t]{7}{*}{ Abruzzi } & \multirow[t]{2}{*}{ Teramo (TE) } & P33, P34, P35D, P36, P38, P39 & 6 & $\mathrm{~V}$ & 2013 \\
\hline & & $\mathrm{P} 32, \mathrm{P} 37 \mathrm{~A} 1, \mathrm{P} 38 \mathrm{~A} 2$ & 3 & $\mathrm{H}$ & \\
\hline & Pianaccio, Valle Castellana (TE) & $\begin{array}{l}\text { L1a, L1c, L3a, L4, L5a, L5b, L6A, } \\
\text { L6b, L7 }\end{array}$ & 9 & $\mathrm{~V}$ & 2014 \\
\hline & \multirow[t]{2}{*}{ Morrice, Valle Castellana (TE) } & $\begin{array}{l}\text { L8, L9A, L9b, L10c, L11, L12a, } \\
\text { L13a, L13b, L15a, L15b }\end{array}$ & 10 & $\mathrm{~V}$ & 2014 \\
\hline & & L14A & 1 & $\mathrm{H}$ & \\
\hline & \multirow[t]{2}{*}{ Valle Castellana (TE) } & B3B & 1 & V & 2016 \\
\hline & & $\mathrm{B} 5 \mathrm{~B}, \mathrm{~B} 6 \mathrm{~B}, \mathrm{~B} 9 \mathrm{~B}$ & 3 & $\mathrm{H}$ & \\
\hline
\end{tabular}


identified by applying a read depth threshold of 50, with these recognized as putative SNPs when present in at least $20 \%$ of the reads (Supplementary Table S1). Out of the 125 nucleotide substitutions detected throughout the CHV-1 genome, 106 (84.8\%) were conservative (pyrimidine to pyrimidine, or purine to purine). In detail, four SNPs were identified outside the coding regions, and of the remaining SNPs, 77 (61.6\%) were synonymous or silent substitutions that did not affect the protein sequence, while 44 resulted in amino acid changes in the ORFA (11) and ORFB (33) polyproteins.

Phylogenetic analysis. The phylogenetic analysis carried out using the MEGA 5 software included the CHV-1 Marche genome (KY471627) in the cluster with the other CHV-1 genomes of European and Asiatic origin, which are phylogenetically different (as demonstrated by the bootstrap values) from the other hypoviruses (i.e., CHV-2, CHV-3, CHV4) that can infect $C$. parasitica (Fig. 2).

\section{Discussion}

Chestnut blight is common in the Marche region, where its incidence ranges from 20 to $60 \%$. The severity of the symptoms appears to be related to the age of the plants, and mostly to the silvicultural

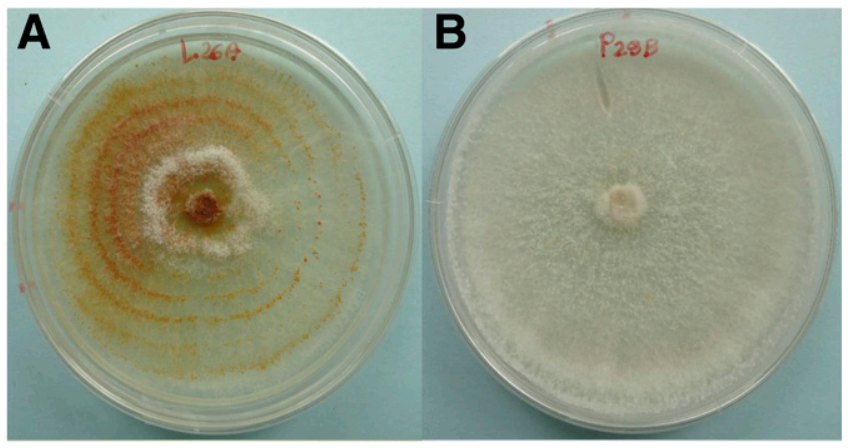

Fig. 1. Representative virulent (A) and hypovirulent (B) colonies of Cryphonectria parasitica isolated during the surveys, cultivated for 15 days with a 16-h photoperiod, at $25^{\circ} \mathrm{C}$. practices adopted (Amorini et al. 2001). In some situations, the severity of decline in chestnut is related to the infestation of the chestnut gall wasp (Meyer et al. 2015). During these surveys carried out from 2013 to 2016, C. parasitica was isolated and identified in about $90 \%$ of the bark samples.

Most of these isolates (i.e., 85 out of 138) were virulent, showing typical morphological features and resulting in wide necrotic areas during the pathogenicity tests. These were isolated from active cankers, on which perithecia were sporadically identified. In Europe, the production of perithecia has been observed in several countries, including Italy, although at medium to low frequency (Prospero and Rigling 2013). On the other hand, 53 hypovirulent $C$. parasitica isolates were obtained and characterized by white mycelia, reduced colony growth, and differentiation of few pycnidia. As demonstrated in previous investigations, these anomalies are caused by viruses that can infect $C$. parasitica, which can induce several phenotypic changes in this fungus, such as a reduction in asexual sporulation, inhibition of sexual reproduction, altered colony morphology, and reduced pigmentation (Nuss 2005). Mycovirus-induced hypovirulence has been reported not only in C. parasitica, but also in Ophiostoma ulmi, O. novo-ulmi, Botrytis cinerea, Sclerotinia sclerotiorum, S. homoeocarpa, and Rosellinia necatrix (Ghabrial et al. 2015).

The $C$. parasitica hypovirulent isolates P16_2, P29B, L25A, and L2A were chosen as representative isolates and next submitted to more detailed molecular characterization. Molecular identification according to the analysis of internal transcribed spacer (ITS) nucleotide sequences confirmed the identity of these fungal isolates. In particular, the ITS region appeared appropriate for molecular identification and to establish the phylogenetic relationships, even with closely related fungal species (Myburg et al. 2004).

To determine the vegetative incompatibility gene profiles of the selected isolates of $C$. parasitica, multilocus PCR assays were carried out as described by Short et al. (2015). This new approach is in agreement with the genetically determined vegetative incompatibility genotypes (Cortesi and Milgroom 1998), and it is generally equivalent to the culture-dependent assays. These culture-dependent assays are time consuming and require well-maintained in-house EU tester strains, and they are not always easy when interpreting

Table 2. Cryphonectria parasitica isolates used in the pathogenicity tests with chestnut cuttings ${ }^{\mathrm{z}}$

\begin{tabular}{|c|c|c|c|}
\hline Isolate & Morphological features & Cortical necrotic area $\left(\right.$ mean $\left.\pm s d ; \mathrm{cm}^{2}\right)$ & Wood necrotic area $\left(\right.$ mean $\left.\pm s d ; \mathrm{cm}^{2}\right)$ \\
\hline $\mathrm{P} 4 \mathrm{C} 4$ & Orange & $7.3 \pm 0.3 \mathrm{a}$ & $10.2 \pm 0.6 \mathrm{a}$ \\
\hline P21A & Orange & $6.5 \pm 1.1 \mathrm{bc}$ & $7.0 \pm 1.3 b$ \\
\hline PD1 & Orange & $5.4 \pm 0.5 \mathrm{de}$ & $6.8 \pm 0.2 b$ \\
\hline P35D & Orange & $6.9 \pm 2 b$ & $6.3 \pm 1.8 \mathrm{~b}$ \\
\hline P31A & Orange & $4.4 \pm 1.1 \mathrm{e}-\mathrm{g}$ & $5.1 \pm 1.4 \mathrm{c}$ \\
\hline P22B & Orange & $5.3 \pm 0.7 \mathrm{~cd}$ & $5.0 \pm 0.5 \mathrm{c}$ \\
\hline $\mathrm{P} 10 \mathrm{C}$ & White & $4.4 \pm 0.6 \mathrm{ef}$ & $4.0 \pm 0.6 \mathrm{~d}$ \\
\hline P14-1 & White & $3.4 \pm 0.3 \mathrm{f}-\mathrm{i}$ & $3.5 \pm 0.5 \mathrm{de}$ \\
\hline $\mathrm{P} 40 \mathrm{~B}$ & White & $3.7 \pm 1.1 \mathrm{f}-\mathrm{h}$ & $3.4 \pm 1 \mathrm{~d}-\mathrm{f}$ \\
\hline $\mathrm{P} 13 \mathrm{~A}$ & White & $2.8 \pm 0.5 \mathrm{f}-1$ & $2.8 \pm 0.8 \mathrm{~d}-\mathrm{g}$ \\
\hline $\mathrm{PC}$ & White & $2.3 \pm 0.51-n$ & $2.7 \pm 0.7 \mathrm{e}-\mathrm{h}$ \\
\hline P16_2 & White & $2.9 \pm 0.2 \mathrm{~g}-1$ & $2.5 \pm 0.1 \mathrm{e}-\mathrm{h}$ \\
\hline $\mathrm{P} 6 \mathrm{C}$ & White & $2.7 \pm 0.8 \mathrm{~h}-\mathrm{m}$ & $2.5 \pm 0.7 \mathrm{e}-\mathrm{h}$ \\
\hline $\mathrm{P} 19 \mathrm{~A}$ & White & $2.6 \pm 0.6 \mathrm{~h}-\mathrm{n}$ & $2.5 \pm 0.7 \mathrm{e}-\mathrm{h}$ \\
\hline $\mathrm{P} 25$ & White & $2.5 \pm 0.2 \mathrm{~h}-\mathrm{n}$ & $2.5 \pm 0.3 \mathrm{e}-\mathrm{h}$ \\
\hline P9 & Orange & $2.6 \pm 1$ h-n & $2.4 \pm 0.8 \mathrm{e}-\mathrm{h}$ \\
\hline P38A2 & White & $2.4 \pm 0.5$ h-n & $2.3 \pm 0.8 \mathrm{f}-\mathrm{h}$ \\
\hline P43 & White & $2.2 \pm 0.1 \mathrm{i}-\mathrm{n}$ & $2.3 \pm 0.5 \mathrm{e}-\mathrm{h}$ \\
\hline P12 & White & $2.6 \pm 0.2 \mathrm{~h}-\mathrm{n}$ & $2.2 \pm 0.3 \mathrm{f}-\mathrm{h}$ \\
\hline P30D & White & $2.5 \pm 0.7 \mathrm{~h}-\mathrm{n}$ & $2.2 \pm 0.6 \mathrm{f}-\mathrm{h}$ \\
\hline P29B & White & $2.4 \pm 0.5$ h-n & $2.1 \pm 0.6 \mathrm{gh}$ \\
\hline $\mathrm{P} 11 \mathrm{C}$ & White & $2.3 \pm 1 \mathrm{i}-\mathrm{n}$ & $2.1 \pm 0.9 \mathrm{gh}$ \\
\hline L25A & White & $2.3 \pm 0.7 \mathrm{i}-\mathrm{n}$ & $2.0 \pm 0.6 \mathrm{gh}$ \\
\hline PA1 & White & $1.8 \pm 0.31-0$ & $1.6 \pm 0.2 \mathrm{hi}$ \\
\hline P41 & White & $1.4 \pm 0.3 \mathrm{n}-\mathrm{o}$ & $1.5 \pm 0.1 \mathrm{hi}$ \\
\hline L2A & White & $1.5 \pm 0.2 \mathrm{~m}-\mathrm{o}$ & $1.4 \pm 0.4 \mathrm{hi}$ \\
\hline
\end{tabular}

${ }^{\mathrm{z}}$ Data followed by different letters are significantly different (Duncan's multiple range tests; $P<0.05$ ). 
barrage reactions (Short et al. 2015). These $C$. parasitica isolates that originated in Marche show four main vegetative incompatibility genotypes that correspond to the vegetative compatibility groups EU12, EU17, EU5, and EU2.

L-dsRNA $(\sim 12 \mathrm{~kb})$ was detected in all the four hypovirulent C. parasitica isolates, which is complete replicative form. M-dsRNA (8 to $10 \mathrm{~kb})$ and S-dsRNA (0.6 to $1.7 \mathrm{~kb}$ ), which represent deleted forms of L-dsRNA that can persist during replication (Montenegro et al. 2008), were not found. To date, mycoviruses have commonly been detected following the isolation of their dsRNA. Today, NGS has become feasible to use as whole-genome and metagenomics (Mokili et al. 2012) approaches, to screen fungi for the presence of potential novel viruses. Recently, Schoebel et al. (2014) detected mycoviruses in the plant pathogenic fungus Hymenoscyphus fraxineus through a combination of NGS with bioinformatics.

The approach used in the present study involved dsRNA isolation and fragmentation, and cDNA library preparation, with Illuminabased sequencing, de novo assembly, and identification of putative sequences of ssRNA and dsRNA virus. This was effective and accurate to determine the virome inside a pool of hypovirulent isolates of C. parasitica.

To the best of our knowledge, this is the first time that the CHV-1 genome has been reconstructed by NGS, and the quality is comparable to that of sequence genomes obtained by the Sanger method and available in the NCBI database. Indeed, Khalifa et al. (2016) recently demonstrated that the accuracy of the sequences obtained using the Illumina platform is comparable with Sanger sequencing analysis. Moreover, to enhance the accuracy of the Illumina analysis, we started from purified dsRNA to maximize the proportion of viral sequences in the data, while using sufficiently rigorous quality controls and maintaining the level of depth and coverage of the sequencing data.

In this situation, we started from a pool of four isolates that shared morphological characteristics and virulence, but were collected from different locations within the Marche region, and only the viral genome of CHV-1 was detected. It is known that $C$. parasitica can be the host of several hypoviruses, reoviruses, and crysoviruses (Ghabrial et al. 2015). From the phylogenetic analysis, it was clear that CHV-1 Marche is in the same cluster with the other CHV-1 genomes available in the NCBI database. In particular, CHV-1 Marche belongs to subtype I, as well as CHV-1/EP721 and CHV-1/Euro 7, whose origin is Italy. It is slightly divergent from CHV-1/EP713 belonging to subtype $\mathrm{F} 1$, whose origin is France, and more consistently different from $\mathrm{CHV}-1 / \mathrm{CN} 280$ belonging to subtype CN6, whose origin is China (Du et al. 2017). On the other hand, CHV-1 Marche is clearly different from other hypoviruses that can infect $C$. parasitica, particularly CHV-2, CHV-3, and CHV-4. It was interesting to observe that $\mathrm{CHV}-2$ is the hypovirus closest to $\mathrm{CHV}-1$ and they have similar genome lengths. Conversely, $\mathrm{CHV}-3$ and $\mathrm{CHV}-4$ are genetically more distant, because they have shorter genomes (by about $9 \mathrm{kbp}$ ) and cause lower degrees of hypovirulence (Ghabrial et al. 2015).

The simplicity of the sample composition here allowed more detailed sequence analysis, which revealed interesting genetic diversity

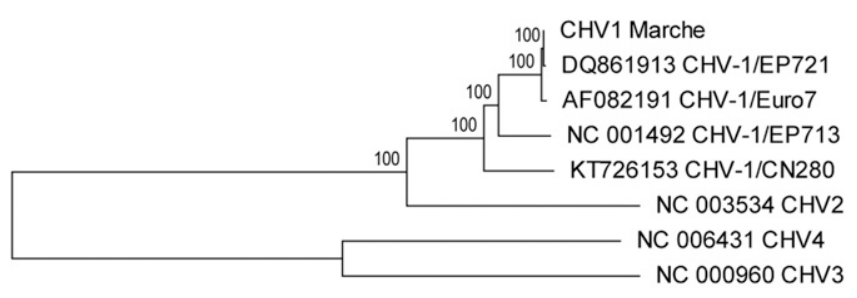

0.1

Fig. 2. Phylogenetic tree constructed from the analysis of the complete genomes of $\mathrm{CHV}-1, \mathrm{CHV}-2$, CHV-3, and CHV-4, as available from GenBank. inside the analyzed pool, although the origin is restricted. A wider genetic diversity was recorded by Feau et al. (2014), who analyzed several samples belonging to different $\mathrm{CHV}-1$ subtypes and whose origins were different. Our study confirmed that in Italy, and in particular the Marche region, CHV-1 subtype I is the only one recorded, as well as in other European countries (Croatia, Greece, Hungary, Macedonia, Slovenia, and Switzerland) (Allemann et al. 1999; Prospero and Rigling 2013). On the other hand, different CHV-1 subtypes were recorded in Spain (D, E, F1, I) (Montenegro et al. 2008; Trapiello et al. 2017) and France (F1, F2, I) (Feau et al. 2014), where a multiple introduction of hypovirulence was hypothesized.

Moreover, in the present study, the genetic diversity is more related to silent substitutions (81), although these also include SNPs (44) causing amino acid substitutions in the two coding regions of the CHV-1 genome (i.e., ORFA, ORFB). The intraspecific variability that was recorded for the pool of four isolates is very common for RNA viruses, which are characterized by complex evolutionary dynamics, high mutation rates, and rapid replication kinetics. Moreover, the presence of silent and non-silent substitutions in ORFA and ORFB might be the result of the interactions between the CHV-1 genome and $C$. parasitica. In particular, viruses have countermeasures to escape host antiviral responses. Many RNA silencing suppressors that target different silencing stages have been reported, and these are diverse in their amino acid sequences and protein structures (Segers et al. 2006). Like pathogenic viruses of mammals, insects, and plants, hypoviruses also encode protein suppressors of RNA interference. The papain-like protease p29 that is encoded by $\mathrm{CHV}-1$ functions as a suppressor of RNA silencing in the natural fungal host (Segers et al. 2006).

In conclusion, the application of NGS is a valid and accurate tool to explore the virome of $C$. parasitica hypovirulent isolates, to analyze the full-length genome of $\mathrm{CHV}-1$, and to exclude the presence of additional ssRNA and dsRNA mycoviruses potentially involved in the hypovirulence.

\section{Acknowledgments}

This study was supported by Marche Polytechnic University through the projects "Diagnosis of emergent disease agents in forest plant" and "Hypovirulence and Cryphonectria parasitica." We thank Prof. Paolo Cortesi and Prof. Michael Milgroom for providing EU testers (EU reference strains). We are grateful to A. Corvaro, L. Marchegiani, A. Moresi, A. Servili, G. Millozzi, G. Girolami, and A. Santini for assistance during the surveys and the collection of samples.

\section{Literature Cited}

Al Rwahnih, M., Daubert, S., Golino, D., and Rowhani, A. 2009. Deep sequencing analysis of RNAs from a grapevine showing Syrah decline symptoms reveals a multiple virus infection that includes a novel virus. Virology 387:395-401.

Allemann, C., Hoegger, P., Heiniger, U., and Rigling, D. 1999. Genetic variation of Cryphonectria hypoviruses (CHV1) in Europe, assessed using restriction fragment length polymorphism (RFLP) markers. Mol. Ecol. 8:843-854.

Amorini, E., Manetti, M. C., Turchetti, T., Sansotta, A., and Villani, F. 2001. Impact of silvicultural system on Cryphonectria parasitica incidence and on genetic variability in a chestnut coppice in Central Italy. For. Ecol. Manage. 142:19-31.

Biraghi, A. 1946. Il cancro del castagno causato da Endothia parasitica. Italia Agric. 7:406.

Bissegger, M., Rigling, D., and Heininger, U. 1997. Population structure and disease development of Cryphonectria parasitica in European chestnut forests in the presence of the natural hypovirulence. Phytopathology 87:50-59.

Bryner, S. F., Prospero, S., and Rigling, D. 2014. Dynamics of Cryphonetria hypovirus infection in chestnut blight cankers. Phytopathology 104:918-925.

Bryner, S. F., Rigling, D., and Brunner, P. C. 2012. Invasion history and demographic pattern of Cryphonectria hypovirus 1 across European populations of the chestnut blight fungus. Ecol. Evol. 2:3227-3241.

Cortesi, P., and Milgroom, M. G. 1998. Genetics of vegetative incompatibility in Cryphonectria parasitica. Appl. Environ. Microbiol. 64:2988-2994.

Du, Y., Lin, Y., Zhou, X., Wang, K., Fang, S., and Deng, Q. 2017. Full-length sequence and genome analysis of CHV1-CN280, a North China isolate of Cryphonectria hypovirus 1 . Arch. Virol. 162:1811-1818.

Dutech, C., Fabreguettes, O., Capdevielle, X., and Robin, C. 2010. Multiple introductions of divergent genetic lineages in an invasive fungal pathogen, Cryphonectria parasitica, in France. Heredity 105:220-228.

Feau, N., Dutech, C., Brusini, J., Rigling, D., and Robin, C. 2014. Multiple introductions and recombination in Cryphonectria hypovirus 1: perspective for a sustainable biological control of chestnut blight. Evol. Appl. 7:580-596. 
Ghabrial, S. A., Castón, J. R., Jiang, D., Nibert, M. L., and Suzuki, N. 2015. 50plus years of fungal viruses. Virology 479-480:356-368.

Grente, J. 1965. Les formes hypovirulentes d'Endothia parasitica et les spoirs de lutte contre le chancre du châtaignier. Comptes-rendus Seances Acad. Agric. Fr. 51:1033-1037.

Heiniger, U., and Rigling, D. 1994. Biological control of chestnut blight in Europe. Annu. Rev. Phytopathol. 32:581-599.

Khalifa, M. E., Varsani, A., Ganley, A. R., and Pearson, M. N. 2016. Comparison of Illumina de novo assembled and Sanger sequenced viral genomes: A case study for RNA viruses recovered from the plant pathogenic fungus Sclerotinia sclerotiorum. Virus Res. 219:51-57.

Liu, Y. C., and Milgroom, M. G. 1996. Correlation between hypovirus transmission and the number of vegetative incompatibility (vic) genes different among isolates from a natural population of Cryphonectria parasitica. Phytopathology 86: 79-86.

MacDonald, W. L., and Fullbright, D. W. 1991. Biological control of chestnut blight: Use and limitations of transmissible hypovirulence. Plant Dis. 75: 656-661

Meyer, J., Gallien, L., and Prospero, S. 2015. Interaction between two invasive organisms on the European chestnut: does the chestnut blight fungus benefit from the presence of the gall wasp? FEMS Microbiol. Ecol. 91:fiv122.

Milgroom, M. G., and Cortesi, P. 2004. Biological control of chestnut blight with hypovirulence: a critical analysis. Annu. Rev. Phytopathol. 42:311338 .

Mokili, J. L., Rohwer, F., and Dutilh, B. E. 2012. Metagenomics and future perspectives in virus discovery. Curr. Opin. Virol. 2:63-77.

Montenegro, D., Aguín, O., Sainz, M., Hermida, M., and Mansilla, J. 2008. Diversity of vegetative compatibility types, distribution of mating types and occurrence of hypovirulence of Cryphonectria parasitica in chestnut stands in NW Spain. For. Ecol. Manage. 256:973-980.

Myburg, H., Gryzenhout, M., Stipes, R. J., and Wingfield, M. J. 2004. Phylogenetic relationships of Cryphonectria and Endothia species, based on DNA sequence data and morphology. Mycologia 96:990-1001.

Nuss, D. L. 1992. Biological control of chestnut blight: An example of virusmediated attenuation of fungal pathogenesis. Microbiol. Rev. 56:561-576.

Nuss, D. L. 2005. Hypovirulence: mycoviruses at the fungal-plant interface. Nat. Rev. Microbiol. 3:632-642.

Prospero, S., and Rigling, D. 2013. Chestnut blight. Pages 318-339 in: Infectious Forest diseases. G. Nicolotti and P. Gonthier, eds. CAB International, Wallingford, U.K.
Rigling, D., and Prospero, S. 2017. Cryphonectria parasitica, the causal agent of chestnut blight: Invasion history, population biology and disease control. Mol. Plant Pathol. doi.org/10.1111/mpp.12542

Robin, C., and Heiniger, U. 2001. Chestnut blight in Europe: diversity of Cryphonectria parasitica, hypovirulence and biocontrol. For. Snow Landsc. Res. 76:361-367.

Schoebel, C. N., Zoller, S., and Rigling, D. 2014. Detection and genetic characterization of novel mycovirus in Hymenoscyphus fraxineus, the causal agent of ash dieback. Infect. Genet. Evol. 28:78-86.

Segers, G. C., Wezel, R. V., Zhang, X., Hong, Y., and Nuss, D. L. 2006. Hypovirus papain-like protease p29 suppresses RNA silencing in the natural fungal host and in a heterologous plant system. Eukaryot. Cell 5:896-904.

Short, D. P., Double, M., Nuss, D. L., Stauder, C. M., MacDonald, W. and Kasson, M. T. 2015. Multilocus PCR assays elucidate vegetative incompatibility gene profiles of Cryphonectria parasitica in the United States. Appl. Environ. Microbiol. 81:5736-5742.

Tamura, K., Peterson, D., Peterson, N., Stecher, G., Nei, M., and Kumar, S. 2011. MEGA5: Molecular Evolutionary Genetics Analysis using maximum likelihood, evolutionary distance, and maximum parsimony methods. Mol. Biol. Evol. 28:2731-2739.

Thompson, J. D., Gibson, T. J., Plewniak, F., Jeanmougin, F., and Higgins, D. G 1997. The CLUSTAL X windows interface: flexible strategies for multiple sequence alignment aided by quality analysis tools. Nucleic Acids Res. 25: 4876-4882.

Trapiello, E., Rigling, D., and Gonzalez, A. J. 2017. Occurrence of hypovirusinfected Cryphonectria parasitica isolates in northern Spain: an encouraging situation for biological control of chestnut blight in Asturian forests. Eur. J. Plant Pathol. 149:503-514.

Varanda, C. M. R., Oliveira, M., Materatski, P., Landum, M., Clara, M. I. E., and Felix, M. R. 2016. Fungal endophytic communities associated to the phyllosphere of grapevine cultivars under different types of management. Fungal Biol. 120:1525-1536.

White, T. J., Bruns, T., Lee, S., and Taylor, J. 1990. Amplification and direct sequencing of fungal ribosomal RNA genes for phylogenetics. Pages 315-322 in: PCR Protocols: a Guide to Methods and Applications. M. A Innis, D. H. Gelfand, J. J. Sninsky, and T. J. White, eds. Academic Press, San Diego, CA.

Zhao, Q. Y., Wang, Y., Kong, Y. M., Luo, D., Li, X., and Hao, P. 2011. Optimizing de novo transcriptome assembly from short-read RNA-Seq data: a comparative study. BMC Bioinformatics 12 (Suppl 14):S2 